\title{
Mapping and Characterization of Two Mutations to Antibiotic Supersusceptibility in Pseudomonas aeruginosa
}

\author{
By BARBARA L. ANGUS, ${ }^{1}$ JANET A. M. FYFE ${ }^{2}$ AND \\ ROBERT E. W. HANCOCK ${ }^{1 *}$ \\ ${ }^{1}$ Department of Microbiology, University of British Columbia, Vancouver, British Columbia, \\ Canada V6T 1 W5 \\ ${ }^{2}$ Department of Bacteriology, University Medical School, Teviot Place, Edinburgh EH8 9AG, UK
}

(Received 6 March 1987; revised 20 May 1987)

\begin{abstract}
Two mutations associated with antibiotic supersusceptibility in Pseudomonas aeruginosa strain Z61 were transferred separately into strain PAO222, using R68.45-mediated conjugation and phage F116L transduction. One mutation $(a b s A)$ was $40 \%$ contransducible with pro- 82 at 26 min on the $P$. aeruginosa chromosome and was associated with increased susceptibility to $\beta$-lactams, gentamicin and hydrophobic agents. Strains carrying the abs $A$ mutation also displayed enhanced uptake of a hydrophobic fluorescent probe, $1-N$-phenylnaphthylamine, and were found, by SDS-PAGE, to be altered in the pattern of lipopolysaccharide O-antigen distribution. The other mutation $(a b s B)$, associated with increased susceptibility to $\beta$-lactams and gentamicin but not to hydrophobic agents, was cotransducible with met-28 and proC at $20 \mathrm{~min}$ on the chromosome. The $a b s B$ mutation caused a structurally undefined alteration in the physical interaction of EDTA and gentamicin with the outer membrane.
\end{abstract}

\section{INTRODUCTION}

The Pseudomonas aeruginosa mutant Z61 (Zimmermann, 1979, 1980) displays dramatically enhanced antibiotic susceptibility (supersusceptibility) when compared to its wild-type parent, K 799. The enhanced susceptibility extends to all types of antibiotics tested and could therefore not be simply explained by a single mutation in an antibiotic-inactivating enzyme nor by an alteration in a specific target molecule. Due to the method of isolation [four mutagenesis steps with ethyl methanesulphonate and one with $N$-methyl- $N^{\prime}$-nitro- $N$-nitrosoguanidine (Zimmermann, 1979)], it was postulated that mutant strain Z61 possessed more than one biochemical alteration leading to antibiotic supersusceptibility (Angus et al., 1982). The diverse outermembrane alterations identified, including changes in lipid A fatty acids (Kropinski et al., 1982) and phosphate (B. L. Angus, unpublished) as well as in the lipopolysaccharide (LPS) core sugars (Kropinski et al., 1982), also indicated multiple mutations. In an attempt to address this question, spontaneous revertants of Z61, which displayed either partial or full reversion to wildtype antibiotic resistance, were isolated (Angus et al., 1982). The isolation of single-step revertants to wild-type indicated to us that there was most likely a single primary mutation in strain Z61 stabilized by one or more other mutations, which may themselves contribute toward antibiotic susceptibility. An alternative genetic approach to the problem was suggested following the successful transfer of two determinants associated with antibiotic supersusceptibility from a clinical isolate of $P$. aeruginosa, strain $492 \mathrm{c}$, into a genetically characterized strain, PAO222, using R68.45-mediated conjugation (Fyfe \& Govan, 1984).

In this paper, we describe the transfer of two mutations associated with antibiotic supersusceptibility from strain Z61 into strain PAO222, the subsequent mapping of the loci by transduction, and further characterization of the associated phenotypes.

Abbreviations: KDO, 3-deoxy-D-mannooctulosonic acid; NPN, 1-N-phenylnaphthylamine. 


\section{Table 1. Pseudomonas aeruginosa strains}

Strain

$$
\text { Genotype }{ }^{*} \text { description }
$$

K799

Z61

Pae K799/WT (ATCC 12055); prototroph

Pae K 799/61, prototrophic: $a b s A$ abs $B$ antibiotic-supersusceptible mutant derived from K 799

Z61 Leu $^{-}$

$\mathrm{PAO} 222$

$\mathrm{Leu}^{-}$mutant of Z61 isolated after EMS treatment

met-28 trp-6 lysA12 his-4 ilv-226 pro-82; wild-type with respect to antibiotic susceptibility

PAZI

met-28 trp-6 lysA12 his-4 ilv-226 absA; antibiotic-susceptible $\mathrm{Pro}^{+}$ transconjugant from cross Z61 $\mathrm{Leu}^{-}(\mathrm{R} 68.45) \times \mathrm{PAO} 222$

PAZ3 lysA12 his-4 ilv-226 pro-82 absB; antibiotic-susceptible $\mathrm{Met}^{+} \mathrm{Trp}^{+}$ transconjugant from cross Z61 Leu $(\mathrm{R} 68.45) \times \mathrm{PAO} 222$

PAZ4
lysA12 his-4 ilv-226 absA absB; antibiotic-susceptible $\mathrm{Pro}^{+}$ transductant of PAZ3 constructed using F116Lc.PAZ1

\section{Reference}

Zimmermann (1979)

Zimmermann (1979)

This paper

Haas \& Holloway (1976)

This paper

This paper

This paper

* Genotype symbols are the same as those used for Escherichia coli; abs $A$ and absB designate enhanced susceptibility to antibiotics as described in this paper. Strains K 799 and Z61 were a gift from W. Zimmermann.

\section{METHODS}

Bacteria and bacteriophages. The bacterial strains used are shown in Table 1. Donor strains carrying the plasmid R68.45 were constructed according to Haas \& Holloway (1976), except in the case of strain Z61, where selection for the plasmid-containing derivative was made on medium supplemented with 50 rather than $500 \mu \mathrm{g}$ carbenicillin $\mathrm{ml}^{-1}$. Phage F116 (Krishnapillai, 1971) was used for transduction and the clear plaque phage mutant F116Lc for strain construction.

Media and culture conditions. The media used in all genetic experiments, nutrient broth, nutrient agar and minimal agar, were previously described (Fyfe \& Govan, 1980). Amino acid supplements were added at a concentration of $1 \mathrm{mM}$. Broth cultures for genetic experiments were grown in McCartney bottles on an orbital incubator at 140 r.p.m. at $37^{\circ} \mathrm{C}$, unless otherwise stated.

For other experiments, proteose peptone no. 2 (Difco; $1 \%$, w/v) was used as a rich medium. Liquid cultures were grown in volumes of $10 \mathrm{ml}$ to 101 in flasks shaken at $37^{\circ} \mathrm{C}$ under conditions of good aeration. Plate cultures were grown on the same media with the addition of $2 \%(w / v)$ agar.

Antibiotic susceptibility testing. Minimal inhibitory concentrations (MICs) were obtained by the agar dilution method. Antibiotics were incorporated at various concentrations into proteose peptone agar plates. Bacteria were grown overnight, diluted 100 -fold and then $0.01 \mathrm{ml}$ bacterial suspension was spotted onto the plates. Plates were grown for $18 \mathrm{~h}$ at $37^{\circ} \mathrm{C}$. MIC values were defined as the lowest concentration of antibiotic which produced inhibition of growth. Gentamicin sulphate, polymyxin B sulphate and trimethoprim were purchased from Sigma; carbenicillin was purchased from Ayerst Laboratories.

Genetic experiments. Auxotrophic mutants were isolated after ethyl methanesulphonate mutagenesis (Watson \& Holloway, 1976) and carbenicillin enrichment (Watson \& Holloway, 1978). Conjugation experiments were performed using the procedure of Fyfe \& Govan (1984), and transductions according to the method of Krishnapillai (1971). Map positions of $a b s A$ and $a b s B$ were determined by co-transduction frequency analysis.

I-N-Phenylnaphthylamine (NPN) uptake assay. This was performed exactly as described by Loh et al. (1984).

Permeabilization of whole cells to lysozyme. Cells were grown to mid-exponential phase in proteose peptone no. 2 medium then centrifuged and resuspended in $5 \mathrm{mM}$-sodium azide (to inhibit respiration), $5 \mathrm{mM} \mathrm{NaHEPES} \mathrm{pH} \mathrm{7.0}$ to an optical density at $600 \mathrm{~nm}$ of approximately 0.5 . Lysozyme was added to a concentration of $50 \mu \mathrm{g} \mathrm{ml}^{-1}$, then various concentrations of the permeabilizing compounds EDTA, gentamicin or polymyxin B were added. Lysis of cells was followed in a Perkin-Elmer Lambda 3 spectrophotometer as decrease in $\mathrm{OD}_{600}$. Microscopic observation was used to check that decrease in $\mathrm{OD}_{600}$ was related to lysis, and controls were performed to ensure that the presence of lysozyme or permeabilizer alone did not cause lysis.

LPS isolation. LPS was isolated from mid-exponential phase cells, using the method of Darveau \& Hancock (1983), extracted with $\mathrm{CHCl}_{3} /$ methanol $(2: 1, \mathrm{v} / \mathrm{v})$ to remove phospholipids and residual SDS, and resuspended in distilled water for further studies.

3-Deoxy-D-mannooctulosonic acid (KDO) assay. KDO was determined by the colorimetric thiobarbituric acid assay of Karkhanis et al. (1978) on samples of LPS hydrolysed in $0.25 \mathrm{M}-\mathrm{H}_{2} \mathrm{SO}_{4}$ for $15 \mathrm{~min}$ at $100^{\circ} \mathrm{C}$. The ammonium salt of KDO (Sigma) was used as the standard.

$S D S-P A G E$. SDS-polyacrylamide gels for the examination of LPS were prepared and run following the method of Lugtenberg et al. (1975) but using $15 \%$ (w/v) acrylamide; $4 \mathrm{M}$-urea was also added (Tsai \& Frasch, 1982) to improve resolution of the heterogeneous chain length O-antigen bands. LPS samples (standardized for KDO 
content) were solubilized by heating at $100{ }^{\circ} \mathrm{C}$ for $5 \mathrm{~min}$ in the presence of $4 \%(\mathrm{w} / \mathrm{v}) \operatorname{SDS}, 10 \%(\mathrm{v} / \mathrm{v}) 2-$ mercaptoethanol, $40 \mathrm{~mm}$-EDTA, $0.125 \mathrm{~mm}$-Tris pH 6.8 and $2 \%(\mathrm{w} / \mathrm{v})$ glycerol. Gels were electrophoresed at $150 \mathrm{~V}$ constant voltage and stained using the LPS silver stain method of Tsai \& Frasch (1982).

Electrophoretic blotting of proteins and LPS. LPS was transferred from polyacrylamide gels to nitrocellulose using the method of Towbin et al. (1979) for SDS gels. Blots were immunostained for specific antigens using the method of Mutharia et al. (1982).

\section{RESULTS}

Transfer of mutations associated with antibiotic supersusceptibility from strain Z61 to strain PAO222

Due to the complex phenotype of Z61, and the number of mutagenesis steps involved in its isolation, we attempted to identify the mutations associated with antibiotic supersusceptibility and to transfer them separately into a common genetic background for further characterization. PAO222 was chosen as a suitable recipient because of its wild-type level of antibiotic susceptibility and range of selectable auxotrophic markers. Before chromosomal transfer between Z61 and PAO222 could take place, it was necessary to construct a donor derivative of Z61. Consequently, a leucine auxotroph of strain Z61 was isolated, and the conjugative plasmid R68.45 was transferred into that strain, resulting in strain Z61 Leu-(R68.45).

Strain Z61 Leu-(R68.45) was mated with strain PAO222 and each of the recipient auxotrophic markers was independently selected. Transconjugants arising were purified and screened for increased susceptibility to cefuroxime on nutrient agar containing $100 \mu \mathrm{g}$ cefuroxime $\mathrm{ml}^{-1}$ (Fyfe \& Govan, 1984). Upon selection for $i l v-226^{+}$, his-4 $4^{+}$or lysA12+, $<1 \%$ of transconjugants demonstrated increased susceptibility to cefuroxime. However, a proportion of the transconjugants selected for the inheritance of pro- $82^{+}$, met $-28^{+}$or $\operatorname{trp}-6^{+}$had coinherited increased susceptibility to cefuroxime. Upon further analysis, these cefuroxime-sensitive transconjugants were found to be of two classes. Those selected as $\mathrm{Pro}^{+}$were supersusceptible (10-40 times more susceptible than PAO222) to carbenicillin, cefoxitin, methicillin, mecillinam, trimethoprim, gentamicin, nalidixic acid, novobiocin and rifampicin, in addition to cefuroxime (Fyfe, 1985). Moderate (two- to tenfold) increases in susceptibility were also noted to flucloxacillin, ampicillin, benzylpenicillin and chloramphenicol. One such transconjugant, designated strain PAZ1, was retained for further characterization. The cefuroxime-sensitive transconjugants selected as $\mathrm{Met}^{+}$and $\mathrm{Trp}^{+}$differed from the strain PAZl class by having wildtype levels of susceptibility to cefoxitin and the hydrophobic or amphiphilic antibiotics trimethoprim, nalidixic acid, rifampicin and chloramphenicol. These conjugants were, in addition, only moderately (fivefold greater than wild-type) susceptible to novobiocin and mecillinam. Both the $\mathrm{Met}^{+}$and $\mathrm{Trp}^{+}$types of transconjugants were identical in this respect and one such transconjugant, PAZ3 $\left(\mathrm{Met}^{+} \mathrm{Trp}^{+}\right.$), was retained for further characterization. Similar matings between a donor derivative of the wild-type strain K 799 and PAO222 did not produce any antibiotic supersusceptible transconjugant.

The antibiotic supersusceptibility associated with strain PAZ1 was apparently due to a mutation (designated $a b s A$ ) which was $40 \%$ contransducible with pro- 82 at 26 min on the PAO chromosome. The mutation associated with antibiotic supersusceptibility in strain PAZ3 (absB) was $9 \%$ cotransducible with met-28 and $19 \%$ cotransducible with proC at $20 \mathrm{~min}$. Fig. 1 shows the locations, on the revised $P$. aeruginosa chromosome map (O'Hoy \& Krishnapillai, 1987), of these and other markers relevant to this study.

A derivative of strain PAZ3, carrying both $a b s A$ and $a b s B$ mutations was also constructed (see Table 1). This strain (PAZ4) showed increased susceptibility to carbenicillin, cefoxitin, methicillin, mecillinam, ampicillin, trimethoprim and novobiocin compared with strain PAZ1, which possessed the $a b s A$ determinant alone.

In Table 2, MIC determinations are presented for three antibiotics, carbenicillin, gentamicin and trimethoprim, considered to be representative of hydrophilic $\beta$-lactams, polycationic aminoglycosides and hydrophobic antibiotics, respectively. As mentioned above, the two types of transconjugants (PAZ1 and PAZ3) could be clearly differentiated by their antibiotic resistance patterns on these three antibiotics. 


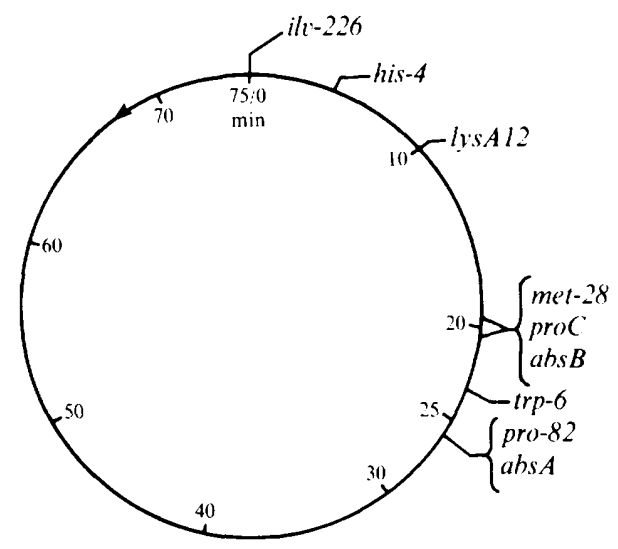

Fig. 1. Genetic map of $P$. aeruginosa (showing only those markers relevant to this study). This recalibrated genetic map is based on the studies of O'Hoy \& Krishnapillai (1987).

Table 2. MIC values of carbenicillin, gentamicin and trimethoprim for P. aeruginosa strains with and without the mutations abs $A$ and $a b s B$

\begin{tabular}{|c|c|c|c|c|}
\hline \multirow[b]{2}{*}{ Strain } & \multirow{2}{*}{$\begin{array}{l}\text { Relevant } \\
\text { mutation(s) }\end{array}$} & \multicolumn{3}{|c|}{$\mathrm{MIC}\left(\mu \mathrm{g} \mathrm{ml}^{-1}\right)$} \\
\hline & & Carbenicillin & Gentamicin & Trimethoprim \\
\hline K799 & $\mathrm{WT}^{*}$ & 20 & 5 & 400 \\
\hline Z61 & $a b s A$ A $a b s B^{\dagger}$ & $<0.25$ & 0.05 & 8 \\
\hline PAO222 & $\mathrm{WT}^{*}$ & 20 & 5 & 400 \\
\hline PAZl & $a b s A$ & 0.25 & $0 \cdot 5$ & 60 \\
\hline PAZ3 & $a b s B$ & 1 & $0 \cdot 1$ & 400 \\
\hline PAZ4 & $a b s A a b s B$ & $0 \cdot 25$ & $0 \cdot 1$ & 20 \\
\hline
\end{tabular}

* WT, wild-type with respect to antibiotic susceptibility.

† Strain Z61 contains additional mutations responsible for antibiotic supersusceptibility.

Table 3. Fluorescence increase due to uptake of NPN in intact cells

Total fluorescence increase was measured 2 min after addition of NPN. Ratios of mutant/wild type for strains PAZ1, PAZ3 and PAZ4 were in comparison with strain PAO222. All results represent the means of three or four trials.

$\begin{array}{lcc}\text { Strain } & \begin{array}{c}\text { Fluorescence increase } \\ \text { (arbitrary units) }\end{array} & \begin{array}{c}\text { Ratio } \\ \text { (mutant/wild type) }\end{array} \\ \text { K799 } & 1.43 & 1.0 \\ \text { Z61 } & 8.47 & 5.9 \\ \text { PAO222 } & 1.03 & 1.0 \\ \text { PAZ1 } & 4.43 & 4.3 \\ \text { PAZ3 } & 0.87 & 0.84 \\ \text { PAZ4 } & 2.80 & 2.7\end{array}$

Outer membrane permeability to the hydrophobic fluorescent probe NPN

Wild-type Gram-negative cells which possess smooth LPS are normally impermeable to hydrophobic substances (Nikaido, 1976). However, since the antibiotic supersusceptible mutant Z61 was known to show increased permeability to hydrophobic substances (Angus, 1986) we investigated our transconjugant strains for defects in the hydrophobic uptake pathway.

The fluorescent probe NPN was used as an indicator of permeability through the hydrophobic portion of the outer membrane, because it fluoresces weakly in an aqueous environment but strongly in a hydrophobic environment, such as in the cell envelope. NPN was 

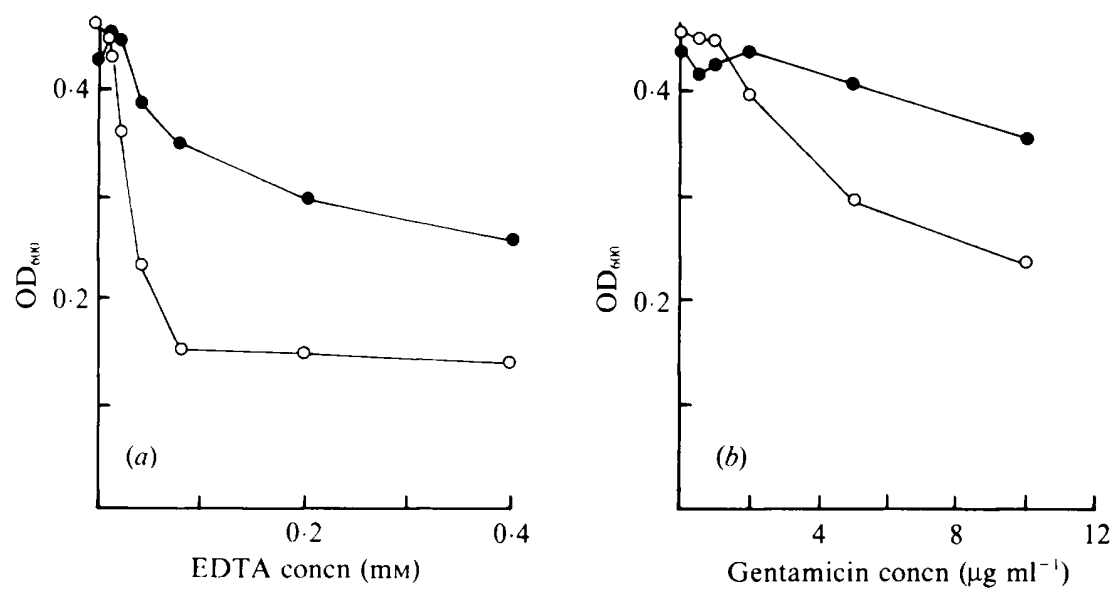

Fig. 2. Permeabilization of whole cells to lysozyme by EDTA $(a)$ or gentamicin (b). EDTA or gentamicin was added to cell suspensions in the presence of lysozyme. Lysozyme-mediated lysis was followed as decrease in $\mathrm{OD}_{600} . \mathrm{O}, \mathrm{K} 799 ; \mathrm{O} 61$.

added to intact cells in buffer, and fluorescence was measured 2 min after addition. As shown previously (Loh et al., 1984; Augus, 1986) NPN was excluded from wild-type cells, but was taken up efficiently by mutant Z61 as demonstrated by enhanced fluorescence emission intensity in the presence of cells of mutant Z61 (Table 3). The transconjugant PAZl also had significantly $(P<0.05$ by Student's $t$-test) increased NPN fluorescence ( 4.3 -fold that of its wildtype parent, PAO222), whereas strain PAZ3 elicited marginally less fluorescence than PAO222. These results agreed well with the MIC results, wherein strain PAZ1 was more susceptible to the relatively hydrophobic antibiotic, trimethoprim. Strain PAZ4, containing both the $a b s A$ and $a b s B$ mutations, also appeared to take up more NPN than the wild-type or the $a b s B$ mutant PAZ3 (Table 3).

\section{EDTA, aminoglycoside and polymyxin B enhancement of lysozyme-mediated cell lysis}

The effect of gentamicin and two other known outer-membrane 'permeabilizers', EDTA and polymyxin B (Warren et al., 1957; Hancock \& Wong, 1984), on permeability of the outer membrane to lysozyme was also examined. A protein such as lysozyme normally would not have access to its target, peptidoglycan on the periplasmic side of the outer membrane, due to the barrier effects of this membrane. Experiments by Hancock et al. (1981) and Hancock \& Wong (1984) showed that certain compounds, particularly polycationic compounds or divalent cation chelators such as EDTA, could aid permeation of lysozyme across the $P$. aeruginosa outermembrane barrier by disrupting membrane integrity. The aminoglycoside antibiotics have been proposed to disrupt outer-membrane integrity by this mechanism (termed self-promoted uptake) during outer-membrane permeation in $P$. aeruginosa. For this reason, permeabilization to lysozyme by gentamicin, polymyxin and EDTA was used to assess alterations in selfpromoted uptake in the antibiotic-supersusceptible strains.

Addition of increasing concentrations of permeabilizing compounds to whole cells, in the presence of lysozyme, caused increasing cell lysis, which was measured spectrophotometrically as decrease in $\mathrm{OD}_{600}$ (Fig. 2). Both EDTA (Fig. 2, Table 4) and polymyxin B (Table 4) caused lysozyme-mediated lysis of between 40 and $85 \%$ of all cells whereas gentamicin was less effective, causing lysis of between 15 and $50 \%$ of cells (Fig. 2, Table 4). Kinetic parameters for the interaction of permeabilizer with the outer membrane could not be examined in this assay since the cell lysis did not follow first-order kinetics. This is compatible with the theory behind self-promoted uptake, wherein interaction of the permeabilizer with the cell surface is cooperative (Loh et al., 1984). Nevertheless we assessed the maximal lysis caused by excess permeabilizer and the concentration of permeabilizer giving $50 \%$ of this maximal lysis $\left(S_{50}\right)$. 
Table 4. Permeabilization of intact cells to lysozyme by EDTA, gentamicin or polymyxin $B$

The effect of the permeabilizers EDTA, gentamicin sulphate (GM) and polymyxin B sulphate (PX) on lysis of whole cells by lysozyme was followed spectrophotometrically as decrease in $\mathrm{OD}_{600}$ in experiments similar to those shown in Fig. 2. $S_{50}$, concentration of permeabilizer causing $50 \%$ maximal lysis.

\begin{tabular}{|c|c|c|c|}
\hline \multirow[b]{2}{*}{ Strain } & \multicolumn{3}{|c|}{ Maximal lysis (\%) } \\
\hline & EDTA & GM & PX \\
\hline K799 & $70 \cdot 0$ & $53 \cdot 0$ & 63 \\
\hline Z61 & $43 \cdot 0$ & $15 \cdot 0$ & 61 . \\
\hline PAO222 & 840 & $42 \cdot 0$ & \\
\hline PAZl & 74.5 & $44 \cdot 0$ & \\
\hline PAZ3 & $85 \cdot 0$ & $20 \cdot 0$ & 57 \\
\hline PAZ4 & $86 \cdot 0$ & $28 \cdot 0$ & $55 \cdot 0$ \\
\hline
\end{tabular}

$\begin{array}{ccc}\overbrace{\text { EDTA }} & \text { GM } & \text { PX } \\ 32.0 & 4.75 & 1.45 \\ 77.0 & 8.86 & 1.70 \\ 15.9 & 14.3 & 1.64 \\ 10.9 & 11.0 & 1.45 \\ 15.0 & 35.4 & 1.82 \\ 9.9 & 22.5 & 2.40\end{array}$

There was little difference between any of the mutant strains and wild-type cells for polymyxin B permeabilization (Table 4), either in the maximum amount of lysis attained or in the $S_{50}$ values. The results for polymyxin permeation correlated well with results obtained from MIC experiments for $P$. aeruginosa grown on polymyxin-containing medium, where there was only a fourfold difference in MIC between strains K799 and Z61 (Angus et al., 1982).

The amount of lysozyme lysis promoted by gentamicin and EDTA permeabilization was substantially lower in the mutant strain Z61 than in the wild-type strain K799, and the concentration of permeabilizer necessary for $50 \%$ maximal lysis was twofold higher (Table 4). With respect to gentamicin only, there was also a marked difference between the $a b s A$ transconjugant PAZ1, which displayed percentage lysis and $S_{50}$ values close to those of the wildtype strain PAO222, and the $a b s B$ transconjugant PAZ3 as well as the $a b s A a b s B$ strain PAZ4, which both had intermediate susceptibilities to permeabilization by gentamicin. The observed differences between antibiotic-supersusceptible mutant Z61 (as well as strains PAZ3 and PAZ4) and the wild-type and $a b s A$ strains indicated that some defect in the interaction of gentamicin at the cell surface existed in strains carrying the $a b s B$ mutation.

\section{SDS-polyacrylamide gel banding pattern of LPS from wild-type and mutant strains}

The LPS of all strains was characterized using SDS-urea-PAGE. LPS in several species (Palva \& Mäkelä, 1980; Darveau \& Hancock, 1983) has been found, by this method, to be heterogeneous with respect to polysaccharide chain length, even within preparations from the same strain. The heterogeneous population of LPS molecules separated roughly according to chain length in this gel system (Peterson et al., 1986) forming a 'ladder'-like pattern, with the largest molecules (rough core substituted with the largest number of $\mathrm{O}$-antigen repeating sugar units) migrating slowest, and rough core with no substitutions migrating fastest. Two major differences between wild-type (K 799) and mutant (Z61) P. aeruginosa LPS were observed by this method (Fig. 3, lanes A and B).

First, the major band with highest mobility, corresponding to rough core LPS, was observed in a different position in the gel for the mutant strain Z61. The higher mobility seen for mutant rough core was consistent with data from a carbohydrate analysis of rough core LPS (K ropinski et al., 1982). There was no difference in rough core LPS migration between any of the recombinant strains, or between the wild-type strains PAO222 and K799. This indicated that the rough core LPS alteration was not due to either the $a b s A$ or $a b s B$ mutations.

The second observation was that there also appeared to be a region of altered LPS banding corresponding to O-antigen-substituted (i.e. smooth) LPS. Two heavily stained groups of bands of lower mobility (Fig. 3), seen in each strain, presumably represented the major chain length species of the heterogeneous LPS population. When gels were loaded at lower concentrations, 

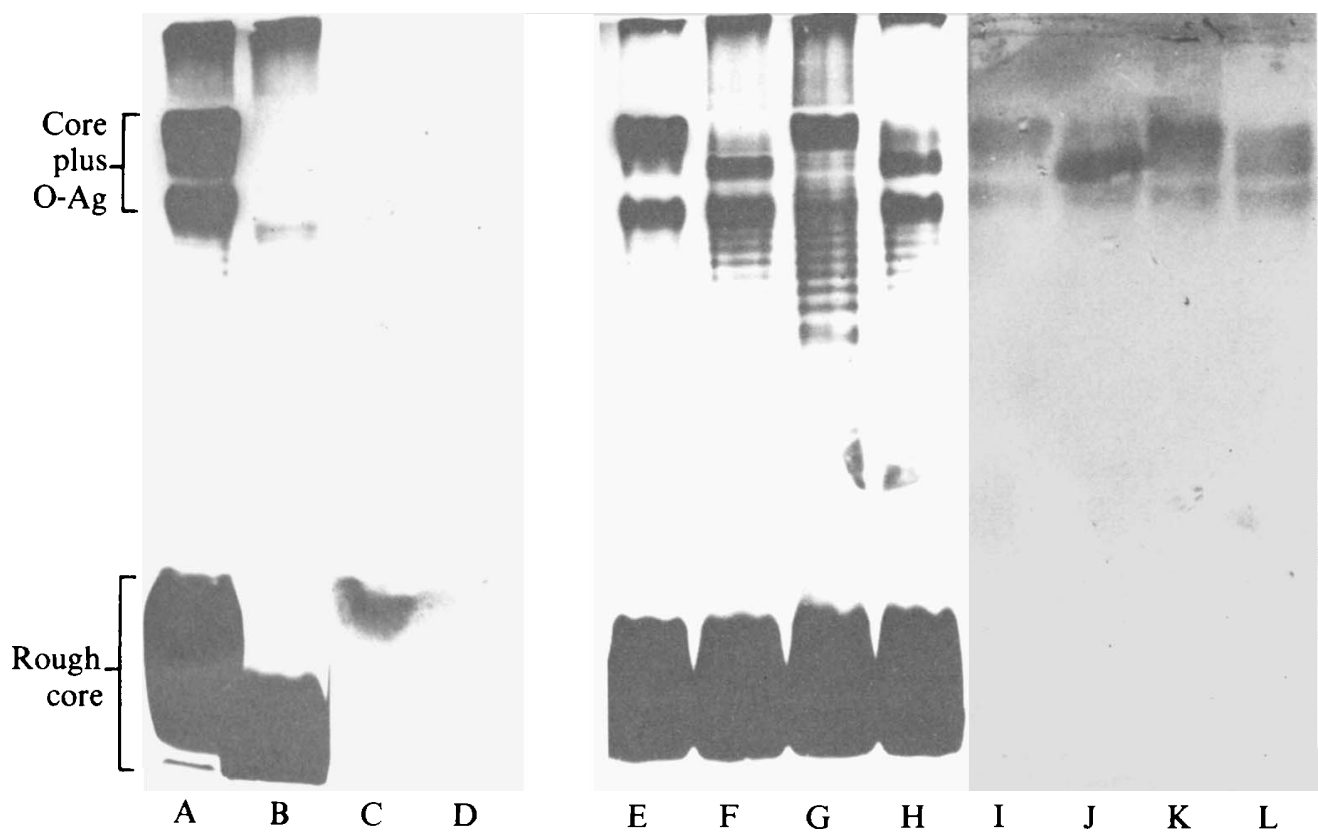

Fig. 3. SDS-polyacrylamide gel banding patterns and immunoreactivity of LPS from wild-type and antibiotic-supersusceptible strains. LPS was isolated as described in Methods, then samples were standardized for KDO content and solubilized in 4\% SDS, 10\% 2-mercaptoethanol, 40 mM-EDTA for $5 \mathrm{~min}$ at $100{ }^{\circ} \mathrm{C}$. Samples were run on a SDS-urea-15\% polyacrylamide gel and stained using periodate plus silver nitrate for carbohydrate (lanes A-B and lanes E-H). Identical samples were blotted onto nitrocellulose and reacted with a monoclonal antibody (MA3-5) specific for $P$. aeruginosa rough core LPS (lanes C-D), or with a monoclonal antibody (MA1-8) specific for $P$. aeruginosa serotype 5 Oantigen (lanes I-L). Lanes: A and C, K799; B and D, Z61 ; E and I, PAO222; F and J, PAZ1 ; G and K, PAZ3; $\mathrm{H}$ and L, PAZ4.

each of these groups of bands was shown to consist of a ladder-like pattern. These groups of bands were well spaced in strains with wild-type (K799 and PAO222: lanes A and E) or the $a b s B$ mutation (PAZ3: lane G). However in mutant Z61, the abs $A$ transconjugant PAZ1 and the $a b s A a b s B$ strain PAZ4 (lanes B, F and $\mathrm{H}$, respectively), the band occupying the upper position in the gel had a higher mobility than the corresponding band in wild-type strains. This phenomenon was reproducibly observed in multiple trials, at various loading concentrations of LPS and over more than one LPS isolation attempt. Since the altered chain length LPS occurred in strains PAZI and PAZ4 (as well as mutant Z61), this indicated that the absA mutation was responsible for the structural property. Mutant Z61 (lane B) was also observed to have fewer rough core molecules substituted with $\mathrm{O}$-antigen chains (shown by the faint staining of lowmobility bands), confirming results from earlier studies (Darveau \& Hancock, 1983).

\section{Immunoreactivity of altered outer-membrane components with monoclonal antibodies directed against LPS}

Although the silver stain method used for the gel in Fig. 3 (lanes A, B and E-H) was purported to be specific for carbohydrate moieties (Tsai \& Frasch, 1982), it was necessary to confirm the identity of the altered components in the mutant as LPS by another criterion. To do this, an electrophoretic blot of LPS from an SDS-urea-polyacrylamide gel (similar to the gel in Fig. 3, lanes A, B and E-H) was made. This was then reacted with monoclonal antibodies raised against LPS determinants from strain PAOl and subsequently with alkaline phosphataseconjugated goat-anti-mouse Fab fragment as the secondary antibody. One of the monoclonal 
antibodes (MA1-8) was O-antigen serotype specific (Hancock et al., 1982) and could only be used for the PAO222-derived strains, PAO222, PAZ1, PAZ3 and PAZ4, and not for the K 799derived strains, $\mathrm{K} 799$ and Z61, for which it had very low reactivity.

The nature of the low-mobility, heavily stained bands from PAO222-derived strains was determined to be LPS, due to their reaction on Western blots (Fig. 3, lanes I-L) with the Oantigen-specific monoclonal antibody MA1-8. The antibody reaction produced a similar pattern to that seen by silver staining of $\mathrm{O}$-antigen, confirming that a population of smooth, $\mathrm{O}$-antigencontaining LPS molecules was altered in strains with the abs $A$ mutation.

The high-mobility stained areas were confirmed to be rough core LPS by reaction on blots (Fig. 3, lanes C and D) with a core-specific monoclonal antibody (MA3-5, Hancock et al., 1983). Monoclonal antibody MA3-5 reacted with LPS core from K799 (Fig. 3, lane C), PAO222, PAZ1, PAZ3 and PAZ4 (data not shown) but not with strain Z61 (Fig. 3, lane D). Perhaps the reason that the core LPS from the mutant strain Z61 did not react with this monoclonal antibody was that the antigenic determinant was present in the outer region of the LPS rough core, and was therefore not present in this mutant (Kropinski et al., 1982).

\section{DISCUSSION}

R68.45-mediated inter-strain crosses have previously been used successfully to identify loci associated with antibiotic susceptibility in $P$. aeruginosa (Fyfe \& Govan, 1984). One of these loci, $b l s A$, associated with supersusceptibility to $\beta$-lactams and various hydrophobic antibiotics in an isolate from a cystic fibrosis patient, was cotransducible with met-28 and proC in the 20 min region of the $P$. aeruginosa chromosome. The $a b s B$ locus identified in this study is close to, but distinct from, bls $A$ (J. Fyfe, unpublished data), and both are close to nalB which codes for resistance to nalidixic acid, novobiocin and various $\beta$-lactams due to decreased permeability to these agents (Rella \& Haas, 1982).

The abs $A$ mutation maps in a different region of the chromosome $(27 \mathrm{~min}$ region) and is responsible for increased susceptibility to 14 antibiotics tested in this study (see text). In addition to the two loci examined, there must therefore be at least one other locus involved in the antibiotic supersusceptibility phenotype of strain Z61, since the $a b s A, B$ double mutant was still less supersusceptible than strain Z61.

Data presented here (Fig. 3) and previously (Kropinski et al., 1982) suggested there were at least two mutations responsible for separate alterations in LPS structure in the antibioticsupersusceptible mutant Z61. One of the mutations, absA, which caused higher mobility of a species of $\mathrm{O}$-antigen-containing LPS, appeared to be directly responsible for wide-spectrum antibiotic supersusceptibility and increased hydrophobic permeability, as seen in the transconjugant PAZ1. In fact, all strains with the higher-mobility O-antigen species (Z61, PAZ1 and PAZ4) took up 2.5- to 6-fold more of the hydrophobic probe NPN (Table 3), and were up to 50 -fold more susceptible to trimethoprim (Table 2), than wild-type strains. This would seem to suggest that possession of the higher-mobility LPS chain-length species associated with the $a b s A$ mutation allowed hydrophobic uptake either by destabilizing the outer membrane in general or by exposing a normally unexposed site on the outer membrane which permitted partitioning of hydrophobic compounds.

$P$. aeruginosa LPS forms a heterogeneous population but, unlike Salmonella LPS, it does not display an even distribution of all O-antigen chain length species (Darveau \& Hancock, 1983). Instead, as shown in this study, two groups of smooth LPS molecules predominate in wild-type strains. During LPS synthesis, long O-antigen chains are first assembled, then transferred intact to rough core acceptor molecules (Osborn, 1979). Since two major distributions of LPS types predominate, it appears that $P$. aeruginosa transfer enzymes, which are involved in transferring completed O-antigen units onto the rough LPS acceptor molecule, are more stringent with respect to O-antigen type than those in $S$. typhimurium. There may be two enzyme variants which recognize different $\mathrm{O}$-antigen chain length species or two rough core species to which different $\mathrm{O}$-antigens are preferentially segregated. This would mean that the $\mathrm{AbsA}^{-}$phenotype might be caused by a mutation in one of these transfer enzymes such that it recognized a shorter 
$\mathrm{O}$-antigen species, or in the rough core composition such that the ability to accept completed $\mathrm{O}$ antigen units from one of the transferases was defective or modified.

The other LPS structural change observed in strain Z61, causing a truncated rough core (Kropinski et al., 1982; Fig. 3) was not observed in the recombinant strains isolated here. This suggested that the increased mobility of the O-antigen LPS species in $a b s A$ strains was not caused by the increased mobility of the rough core LPS. The structural change associated with the $a b s B$ mutation, which caused $\beta$-lactam and aminoglycoside susceptibility and partial disturbance of self-promoted uptake, was not apparent from this study. The low permeabilization to lysozyme of the outer membrane of strains PAZ1 and Z61 by gentamicin and EDTA would seem to indicate that this strain possessed an alteration in its LPS $\mathrm{Mg}^{2+}$-binding sites, which are important for stabilizing the surface of the outer membrane through crossbridging of adjacent LPS molecules. Such an alteration was recently observed in studies of strain Z61, using a cationic spin label probe (Peterson et al., 1985). We are currently investigating the possibility that this alteration is associated with the $a b s B$ mutation.

In conclusion, our data indicate that the LPS of $P$. aeruginosa plays a role in determining the extent of outer membrane permeability and consequently in the high antibiotic resistance of this organism.

Financial assistance from the Canadian Cystic Fibrosis Foundation and BC Health Care Research Foundation (to R.E.W.H.) and the Scottish Hospital Endowment Research Trust (to J.A.M.F.) is gratefully acknowledged.

\section{REFERENCES}

ANGUS, B. L. (1986). Structural and functional studies on the role of the outer membrane of Pseudomonas aeruginosa in resistance and permeability to antibiotics. $\mathrm{PhD}$ thesis, University of British Columbia.

Angus, B. L., Carey, A. M., Caron, D. A., Kropinski, A. M. \& HANCOCK, R. E. W. (1982) Outer membrane permeability in Pseudomonas aeruginosa: comparison of a wild type strain with an antibiotic supersusceptible mutant. Antimicrobial Agents and Chemotherapy 21, 299-309.

Darveau, R. P. \& Hancock, R. E. W. (1983). Procedure for isolation of bacterial lipopolysaccharides from both smooth and rough Pseudomonas aeruginosa and Salmonella typhimurium strains. Journal of Bacteriology 155, 831-838.

FYFE, J. A. M. (1985). Antibiotic supersensitive mutants of Pseudomonas aeruginosa. $\mathrm{PhD}$ thesis, University of Edinburgh.

Fyfe, J. A. M. \& Govan, J. R. W. (1980). Alginate synthesis in mucoid Pseudomonas aeruginosa: a chromosomal locus involved in control. Journal of General Microbiology 119, 443-450.

Fyfe, J. A. M. \& Govan, J. R. W. (1984). Chromosomal loci associated with antibiotic hypersensitivity in pulmonary isolates of Pseudomonas aeruginosa. Journal of General Microbiology 130, 825-834.

HaAs, D. \& Holloway, B. W. (1976). R factor variants with enhanced sex factor activity in Pseudomonas aeruginosa. Molecular and General Genetics 144, 243251.

Hancock, R. E. W. \& Wong, P. G. W. (1984). Compounds which increase the permeability of the Pseudomonas aeruginosa outer membrane. Antimicrobial Agents and Chemotherapy 26, 48-52.

Hancock, R. E. W., Raffle, V. J. \& Nicas, T. I. (1981). Involvement of the outer membrane in gentamicin and streptomycin uptake and killing in Pseudomonas aeruginosa. Antimicrobial Agents and Chemotherapy 19, 777-783.
Hancock, R. E. W., Wieczorek, A. A., Mutharia, L. M. \& PoOle, K. (1982). Monoclonal antibodies against Pseudomonas aeruginosa outer membrane antigens: isolation and characterisation. Infection and Immunity 37, 166-171.

Hancock, R. E. W., Mutharia, L. M., Chan, L., Darveau, R. P., Speert, D. P. \& Pier, G. B. (1983). Pseudomonas aeruginosa isolates from patients with cystic fibrosis: a class of serum-sensitive, nontypable strains deficient in lipopolysaccharide $\mathrm{O}$ side chains. Infection and Immunity 42, 170-177.

KaRkhanis, Y. D., ZeltNeR, J. Y., JACKSON, J. J. \& CaRlo, D. J. (1978). A new and improved microassay to determine 2-keto-3-deoxyoctonate in lipopolysaccharide of Gram negative bacteria. Analytical Biochemistry 85, 595-601.

KRISHNAPILlAI, V. (1971). A novel transducing phage: its role in recognition of a possible new host controlled modification system in Pseudomonas aeruginosa. Molecular and General Genetics 114, 134143.

Kropinski, A. M., Kuzio, J., ANGus, B. L. \& HANCOCK, R. E. W. (1982). Chemical and chromatographic analysis of lipopolysaccharide from an antibiotic supersusceptible mutant of Pseudomonas aeruginosa. Antimicrobial Agents and Chemotherapy 21, 310-319.

LoH, B., Grant, C. \& Hancock, R. E. W. (1984). Use of the fluorescent probe $1-N$-phenylnaphthylamine to study the interactions of aminoglycoside antibiotics with the outer membrane of Pseudomonas aeruginosa. Antimicrobial Agents and Chemotherapy 26, 546-551.

Lugtenberg, B., Meijers, J., Peters, R., van Der HoCK, P. \& VAN ALPHEN, L. (1975). Electrophoretic resolution of the 'major outer membrane protein' of Escherichia coli $\mathrm{K}-12$ into four bands. FEBS Letters 58, 254-258.

Mutharia, L. M., Nicas, T. I. \& Hancock, R. E. W. 
(1982). Outer membrane proteins of Pseudomonas aeruginosa serotype strains. Journal of Infectious Diseases 146, $770-779$.

NikaIDO, H. (1976). Outer membrane of Salmonella typhimurium. Transmembrane diffusion of some hydrophobic substances. Biochimica et biophysica acta 433, 118-132.

O'HoY, K. \& KRISHNAPIllaI, V. (1987). Recalibration of the Pseudomonas aeruginosa genetic map using high frequency of recombination donors. Plasmid (in the Press).

OSBORN, M. J. (1979). Biosynthesis and assembly of the LPS of the outer membrane. In Bacterial Outer Membranes: Biogenesis and Functions, pp. 15-34. Edited by $\mathrm{M}$. Inouye. London: Wiley-Interscience.

Palva, E. T. \& MäKELÄ, P. H. (1980). LPS heterogeneity in Salmonella typhimurium analysed by SDSPAGE. European Journal of Biochemistry 107, 137143.

Peterson, A. A., Hancock, R. E. W. \& McGroarty, E. J. (1985). Binding of polycationic antibiotics and polyamines to lipopolysaccharides of Pseudomonas aeruginosa. Journal of Bacteriology 164, 1256-1261.

Peterson, A. A., Haug, A. \& McGroarty, E. J. (1986). Physical properties of short- and long-Oantigen-containing fractions of LPS from Escherichia coli O111:B4. Journal of Bacteriology 165, 116 122.

Rella, M. \& HaAs, D. (1982). Resistance of $P$. aeruginosa PAO to nalidixic acid and low levels of beta-lactam antibiotics: mapping of chromosomal genes. Antimicrobial Agents and Chemotherapy 22, 242-249.

Towbin, M., Staehelin, T. \& Gordon, J. (1979). Electrophoretic transfer of proteins from polyacrylamide gels to nitrocellulose sheets: procedure and some applications. Proceedings of the National Academy of Sciences of the United States of America 76, 4350-4354.

Tsai, C.-M. \& Frasch, C. E. (1982). A sensitive silver stain for detecting lipopolysaccharides in polyacrylamide gels. Analytical Biochemistry 119, 115-119.

Watson, J. M. \& Holloway, B. W. (1976). Suppressor mutations in Pseudomonas aeruginosa. Journal of Bacteriology 125, 780-786.

Watson, J. M. \& Holloway, B. W. (1978). Linkage map of Pseudomonas aeruginosa PAT. Journal of Bacteriology 136, 507-521.

Warren, G. H., Gray, J. \& Yurchenko, J. A. (1957). Effect of polymyxin on the lysis of Neisseria catarrhalis by lysozyme. Journal of Bacteriology 74, 788-793.

ZimmermanN, W. (1979). Penetration through the Gram-negative cell wall: a co-determinant of the efficacy of beta-lactam antibiotics. International Journal of Clinical Pharmacology and Biopharmacology 17, 131-134.

ZimmermanN, W. (1980). Penetration of beta-lactam antibiotics into their target enzymes in Pseudomonas aeruginosa : comparison of a highly sensitive mutant with its parent strain. Antimicrobial Agents and Chemotherapy 18, 94-100. 